\title{
NOTES
}

\section{COMPRESSING A CHESS-ENDGAME DATABASE}

\author{
Christopher A. Marris
}

Hull, England

ABSTRACT

\begin{abstract}
A method of compressing a chess-endgame database taking advantage of the arrangement of the number of moves-to-mate between adjacent positions within the database is described. A subset of KQK with White to move (WTM) is used to illustrate the technique.
\end{abstract}

\section{INTRODUCTION}

One of the problems with computer-chess endgame databases (especially when microcomputer-implemented) is their large size. To show how such databases may profitably be compressed considerably, we take as an example a subset of the KQK endgame. The subset considered has fixed positions for the two Kings (WK on e8; BK on a8). Diagram 1 shows the number of moves to mate for each of the 45 legal positions.

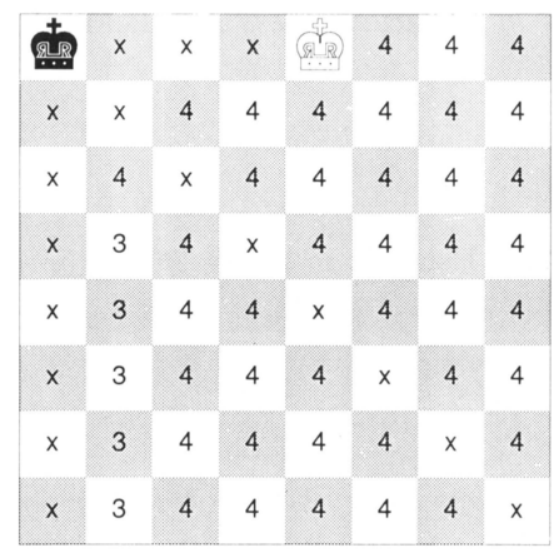

DIAGRAM 1

$X=$ forbidden position of the white Queen.

We number the rows and files from 0 to 7 , and use the following abbreviations: WKR $=\operatorname{row}(\mathrm{WK})$; WKF $=$ file $(W K) ; B K R=\operatorname{row}(B K) ; B K F=$ file $(B K) ; W Q R=\operatorname{row}(W Q) ; W Q F=$ file(WQ). A possible indexing function $\mathrm{F} 1$ for each $\mathrm{KQK}$ position may now be:

$$
\mathrm{F} 1=32768 * \mathrm{BKR}+4096 * \mathrm{BKF}+512 * \mathrm{WKR}+64 * \mathrm{WKF}+8 * \mathrm{WQR}+\mathrm{WQF} .
$$

This indexing method guarantees that each position in the KQK database has a different entry point in the database, given by F1. 


\section{DIFFERENT INDEXING FUNCTIONS}

The subset of moves-to-mate (M) of the KQK database, corresponding with the white King and black King confined to positions $\mathrm{e} 8$ and a8, respectively, using F1 as indexing function, is given in Figure 1 (values of 0 indicate illegitimate $\mathrm{KQK}$ positions, otherwise moves to mate).

entry[233216] entry[233279] $\downarrow$

0344444003444404034440440344044403404444040444440044444400000444

Figure 1: Moves-to-mate (M) using F1 as the indexing function.

If we define an M-interval as a contiguous set of database entries with the same M-values (disregarding illegitimate positions), the information in Figure 1 may equally well be expressed by Table 1.

\begin{tabular}{|r|l|l|c|}
\hline \multicolumn{3}{|c|}{ M-interval } & \multirow{2}{*}{ M-value } \\
\hline$\#$ & lower bound & upper bound & \\
\hline 1 & 233216 & 233217 & 3 \\
2 & 233218 & 233222 & 4 \\
3 & 233223 & 233225 & 3 \\
4 & 233226 & 233231 & 4 \\
5 & 233232 & 233233 & 3 \\
6 & 233234 & 233239 & 4 \\
7 & 233240 & 233241 & 3 \\
8 & 233242 & 233247 & 4 \\
9 & 233248 & 233249 & 3 \\
10 & 233250 & 233279 & 4 \\
\hline
\end{tabular}

Table 1: M-intervals corresponding to Figure 1.

Note that by convention we include illegitimate positions as part of the next-higher indexed legitimate position.

That the indexing function may have a profound influence on the number of intervals generated is shown by exhibiting the alternative indexing function F2:

$$
\mathrm{F} 2=32768 * \mathrm{BKR}+4096 * \mathrm{BKF}+512 * \mathrm{WKR}+64 * \mathrm{WKF}+8 * \mathrm{WQF}+\mathrm{WQR} .
$$

The above procedure is repeated to obtain Figure 2 and Table 2.

entry[233216]. entry[233279] $\downarrow$

0000000033333400444440404444044044404440440444444044444404444444

Figure 2: Moves-to-mate (M) using F2 as the indexing function. 


\begin{tabular}{|l|l|l|c|}
\hline \multicolumn{3}{|c|}{ M-interval } & \multirow{2}{*}{ M-value } \\
\hline$\#$ & lower bound & upper bound & \\
\hline 1 & 233216 & 233228 & 3 \\
2 & 233229 & 233279 & 4 \\
\hline
\end{tabular}

Table 2: M-intervals corresponding to Figure 2.

So, whereas indexing function $\mathrm{F} 1$ leads to $10 \mathrm{M}$-intervals, $\mathrm{F} 2$ reduces this figure (by effectively rearranging the KQK subset database) to an optimal value of 2. (Optimal because the number of M-intervals equals the number of different M-values.)

Other indexing functions may be used. The number of different indexing functions of the kind of F1 and F2 equals 720 (being 6!, i.e., all permutations of the 6 parameters (BKR, BKF, etc.) involved), but of course also other types of indexing functions may be tried.

For the complete KQK database with WTM the maximum number of moves-to-mate is known to be 10 . It is tempting to devise an indexing function yielding, for the complete $\mathrm{KQK}$ database, a number of M-intervals which exceeds the required minimum of 10 by the lowest amount possible.

\section{REFERENCES}

Held, G. (1983). Data Compression. Wiley/Heyden. ISBN 0471 26248X.

Hewlett Jr., C.W. (1977). Chess Catalog for the Endgame White Queen and White King vs. Black King. Publ. by C.W. Hewlett, Waynesboro, Virginia, USA.

Roycroft, A.J. (1985). Chess-Endgame Data-Base 'Oracles': Necessary and Desirable Features. ICCA Journal, Vol. 8, No. 2, pp. 100-104.

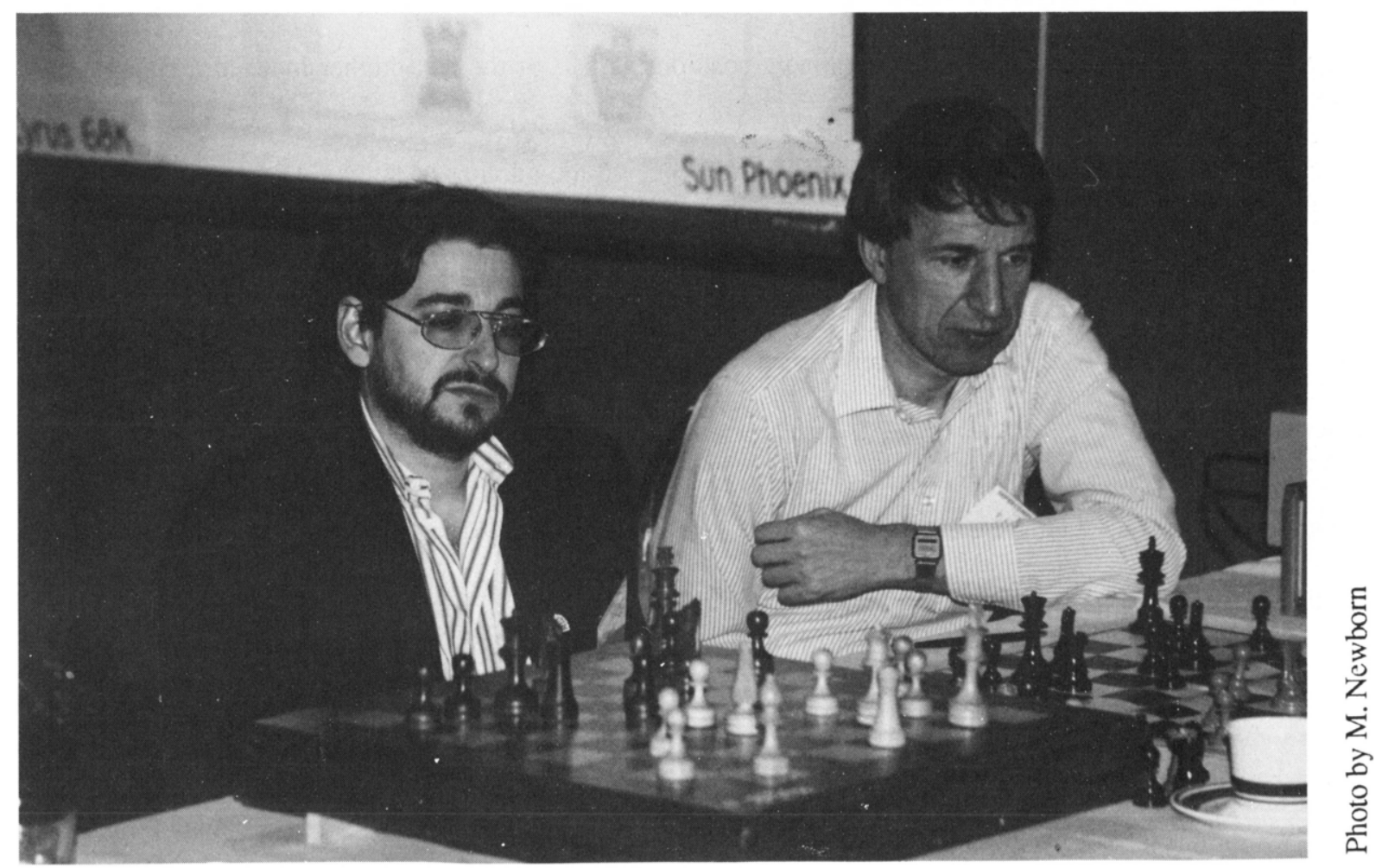

Ossi Weiner, suitably coloured, playing Mephistophelian gambits.

Tony Marsland has the role of Dr. Faustus. ACM Tournament Orlando, Florida, 1988. 